\title{
Topical preventive measures to avoid or reverse white spot lesions in orthodontic patients
}

\author{
Abstracted from \\ Bergstrand F, Twetman S. \\ A review on prevention and treatment of post-orthodontic white spot lesions - \\ evidence-based methods and emerging technologies. Open Dent J 2011; 5: 158-162. \\ Address for correspondence: Svante Twetman, Department of Odontology, \\ Faculty of Health Sciences, University of Copenhagen, Nørre Allé 20, \\ DK-2200 Copenhagen N, Denmark. E-mail: stwe@sund.ku.dk
}

\section{Question: What are the current and emerging technologies to prevent and reverse post-orthodontic white spot lesion (WSL)?}

\begin{abstract}
Data sources Medline/PubMed and the Cochrane Library.
\end{abstract}
Study selection Only human controlled in vivo studies of topical technologies with white spot lesions incidence/reversals or other relevant surrogate endpoints in the English language were accepted. Data extraction and synthesis Data on design, performance and outcome were extracted independently but no formal quality grading was conducted. The prevented WSL fraction was calculated as the difference in mean WSL increment between the intervention and control groups, expressed as percentage of the increment in the control group. For WSL reversals, the absolute risk reduction (ARR\%) was calculated when possible.

Results Twenty-five studies were included; nine considered primary prevention, eight WSL reversals while six utilised surrogate measures such as bacterial counts, plaque amount or $\mathrm{pH}$-values. Fluoride use was the most common intervention (nine papers), followed by antibacterial agents (five papers), use of casein phosphopeptide-stabilised amorphous calcium phosphate (CPP-ACP) for remineralisation (five papers) and various other methods (six papers). The mean prevented fraction based on six topical fluoride trials was $42.5 \%$ with a range from $-4 \%$ to $73 \%$.

Conclusions The recent papers provided the strongest support for regular professional applications of fluoride varnish around the bracket base during the course of orthodontic treatment. For the treatment of post-orthodontic WSL, home-care applications of a remineralising cream, based on CPP-ACP, as adjunct to fluoride toothpaste could be beneficial but the findings were equivocal. Only studies with surrogate endpoints are available for technologies such as sugar alcohols and probiotics. Further well-designed studies with standardised regimes and endpoints are needed before guidelines on the non-fluoride technologies can be recommended.

\section{Commentary}

Orthodontic treatments with fixed appliances may increase the risk for the development of white-spot lesions (WSL). There are various topical preventive measures which have been proposed to minimise the incidence and severity of WSL. A Cochrane review in 2004 evaluated the effects of fluorides for the primary prevention of WSL during fixed-appliance orthodontic treatment. This review found almost no evidence that daily fluoride mouthrinses could reduce incidence of WSL around fixed appliances. ${ }^{1}$ Recently, some clinical studies have investigated the effect of treatments, mainly topical creams with casein phosphopeptide-stabilised amorphous calcium phosphate (CCP-ACP), on the control of existing WSLs after debonding fixed orthodontic appliances.

The aims of the present review (Bergstrand \& Twetman, 2011) were to update the evidence on current methods used to prevent WSLs during orthodontic treatments and to review the evidence on emerging treatments proposed to reverse WSL after debonding. Controlled clinical trials published between 2004 and 2011 were searched and 25 studies were included. For primary prevention, nine studies were retrieved: seven evaluated the effect of topical fluorides. For secondary prevention, nine studies were found and five concerned the effect of CCP-ACP. The main outcome was the difference in the progression (Prevented fraction) or the regression (Absolute Risk Reduction) of the WSL between study groups. Six studies were considered apart as they used surrogate measures. Data were analysed descriptively and no meta-analysis was conducted. The authors concluded that the findings confirm the use of regularly professionally applied fluoride varnish around the brackets to prevent WSL. They also considered that CPP-ACP as an adjunct to fluoride toothpastes could be beneficial but they indicated that the level of evidence is poor.

This review cannot be considered as a systematic review, as clearly stated by the authors themselves. The search was limited to a short period (2004-2011) and only considered studies written in English. The reader must keep those limitations in mind when reading the review. There is here a potential publication bias as some clinical studies might have been published before 2004 or written using other languages.

The review pursued two different aims and was not focused on the evaluation of the efficacy of one type of treatment. It explored the effect of topical fluorides and CCP-ACP, but also of ozone or sealants. Those multiple objectives have complicated the analysis, as the number of comparisons needed was multiplied. It must also be noted that some of the included studies compared the treatment 
group to a placebo/no treatment group while in other studies, one treatment was compared with another. Analysing data from these two types of design together seems inappropriate given that the ranges of the between-group differences may be higher when the reference group receives no effective treatment.

Within one comparison (primary or secondary prevention), different types of outcomes were mixed, including scores obtained with a clinical evaluation or micro-hardness, Quantitative Light Fluorescence (QLF) and decalcification measures. The way the evolution scores (regression or incidence) have been calculated was not given. The criteria and procedures used to evaluate clinically the WSL were not described. Thus the reliability and validity of the data were not ensured. This is an important issue. It is well known that the detection of initial carious lesions on smooth surfaces relies on the visual appreciation of slight changes in enamel's colour or brightness. Thus, clinical data obtained using standardised and well-defined criteria such as the International Classification and Assessment System (ICDAS), which was used in the study of Bailey et al. (2009), should be preferred. ${ }^{2}$

The authors stated that no formal quality grading was done. In the results, they have indicated only the type of design used, namely randomised, non-randomised clinical trial or split mouth design. Some studies were designed in a way that made double blinding possible, but no information is given in the review. Concerning the other studies, the way examiners were kept blind to the patients' groups is not described nor discussed. The drop-out proportion for each survey is unknown; the authors mentioned in the discussion that 'few drop outs' were reported. It was stated that studies were of 'mixed quality' but the reader cannot appreciate objectively the studies' quality. This quality evaluation should have been done and summarised in a table in the results section.

When possible, the prevented fraction (PF) or the absolute risk reduction (ARR) were calculated. The significance of the difference observed between groups was reported but no confidence interval (CI) was calculated. It would have been useful, even if the results had been presented in a descriptive manner, to have the PF and ARR values with their CIs. This would have improved the quality of the review, harmonised the presentation of the data and helped the reader to appreciate the importance of each study.

In Table 2, three randomised clinical studies (RCT) were compared, characterised by different lengths of follow-up. They all evaluated the effect of a CPP-ACP remineralising cream on the clinical regression of WSL after debonding. One RCT evaluated the effect of a CPP-ACP versus placebo during 4, 8 and 12 weeks. There was no statistical difference in the proportion of WSL, which regressed after 4 , and 8 weeks. After 12 weeks, a slight difference was observed with an Odds Ratio of 2.33 (1.06; 5.14). ${ }^{2}$ Another RCT evaluated the effect of a 3-months application of CPP-ACP versus $\mathrm{NaF}$ rinse with a follow up of 12 months. The ACP-CCP cream was found more effective in reducing the number of WSL after 12 months. $^{3}$ Another RCT studied the effect of a CPP-ACP cream but no significant difference was noticed after 4 weeks of follow up. ${ }^{4}$ This example shows that studies conducted with different lengths of follow up need to be compared with caution. This important methodological aspect was only mentioned in the discussion section of the review.

In conclusion, this review gives a general insight on studies that evaluated the topical preventive measures used to avoid or reverse WSL in orthodontic or post-orthodontic patients. In order to have a complete evaluation of the efficacy of topical fluorides for the primary prevention of WSL, I would suggest waiting for the update of the 2004 Cochrane review. The efficacy of ACP-CCP in reversing WSL for post-orthodontic patients, as stated by the authors of the present review, needs to be confirmed by other large, well-conducted randomised clinical studies. The clinical criteria used to evaluate the regression of WSLs, the endpoint chosen and the type of comparison made (vs placebo or vs other treatment) would be key points for the future studies and reviews.

Stéphanie Tubert-Jeannin

Department of Dental Public Health, Faculty of Dental Surgery, Universite d'Auvergne, CHU de Clermont-Ferrand, France

1. Benson PE, Parkin N, Millett DT, Dyer FE, Vine S, Shah A. Fluorides for the prevention of white spots on teeth during fixed brace treatment. Cochrane Database Syst Rev. 2004; Issue 3. Art. No. CD003809.

2. Bailey DL, Adams GG, Tsao CE, et al. Regression of post-orthodontic lesions by a remineralizing cream. / Dent Res 2009; 88:1148-1153. Epub 2009 Nov 3.

3. Andersson A, Sköld-Larsson K, Hallgren A, Petersson LG, Twetman S. Effect of a dental cream containing amorphous cream phosphate complexes on white spot lesion regression assessed by laser fluorescence. Oral Health Prev Dent 2007; 5: 229-233.

4. Bröchner A, Christensen C, Kristensen B, et al. Treatment of post-orthodontic white spot lesions with casein phosphopeptide-stabilised amorphous calcium phosphate. Clin Oral Investig. 2011; 15: 369-373. Epub 2010 Apr 10.

Evidence-Based Dentistry (2012) 13, 21-22. doi:10.1038/sj.ebd.6400844 